\title{
Editor's Perspective on 4
}

\section{Functional imaging of cerebral arteriovenous malformations with a comment on cortical} reorganization. Richard Leblanc, Ernst Meyer, Robert Zatorre, Denise Klein, and Alan Evans

This paper is interesting in its use of positron emission tomography (PET) scanning combined with anatomical imaging. The authors present a major challenge to those attempting to use functional magnetic resonance imaging for functional evaluation. The paper is weakened by lack of correlation of the PET findings with intraoperative cortical stimulation. Furthermore, the authors' emphasis on two major speech areas in the classic Broca's and Wernicke's areas is likely too simplistic. It is clear that significant variation in speech localization exists, especially in the presence of cortical pathology. Further activated areas will vary quite dramatically with the particular speech task being assessed. Similarly it is unclear why only the SI cortex was demonstrated during sensory stimulation, when there is known prominent activation of areas in addition to SI, for example SII and the retroinsular parietal operculum. The editor is not convinced that the juxtaposition of "eloquent cortex" and an arteriovenous malformation precludes its surgical treatment, although this knowledge can certainly help make appropriate preoperative and intraoperative decisions. 